\title{
Two new species of Cladosporium from leaf spots of Paris polyphylla in north-western Yunnan Province, China
}

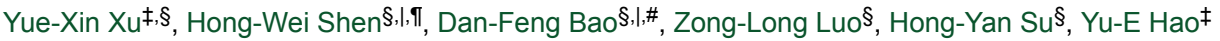 \\ ‡ College of Public Health, University of South China, Hengyang, China \\ $\S$ College of Agriculture and Biological Sciences, Dali University, Dali, China \\ I Center of Excellence in Fungal Research, Mae Fah Luang University, Chiang Rai, Thailand \\ I School of Science, Mae Fah Luang University, Chiang Rai, Thailand \\ \# Department of Entomology and Plant Pathology, Faculty of Agriculture, Chiang Mai University, Chiang Mai, Thailand
}

Corresponding author: Hong-Yan Su (suhongyan16@163.com), Yu-E Hao (haohao200701@126.com)

Academic editor: Ning Jiang

Received: 28 Oct 2021 | Accepted: 14 Dec 2021 | Published: 23 Dec 2021

Citation: Xu Y-X, Shen H-W, Bao D-F, Luo Z-L, Su H-Y, Hao Y-E (2021) Two new species of Cladosporium from leaf spots of Paris polyphylla in north-western Yunnan Province, China. Biodiversity Data Journal 9: e77224. https://doi.org/10.3897/BDJ.9.e77224

\section{Abstract}

\section{Background}

During the survey of pathogenic fungi on medicinal plant leaves in Yunnan Province, China, two Cladosporium-like taxa were isolated from leaf spots of Paris polyphylla. Based on morphological characteristics and phylogenetic analysis of combined ITS, TEF1- $\alpha$ and ACT genes, two new species were discovered.

\section{New information}

Two new species Cladosporium yunnanensis and C. paris are introduced, the detailed descriptions and illustrations are provided. Morphology of the two new species is compared with other related Cladosporium species. This study widens the host diversity of the genus Cladosporium. 


\section{Keywords}

asexual morph, Cladosporium, hyphomycetes, phylogeny, taxonomy

\section{Introduction}

Cladosporium is one of the largest and most heterogeneous genera of hyphomycetous fungi (Dugan et al. 2004). It was initially described by Persoon (1794) from rotten wood as Dematium herbarum Pers., which was later synonymised by Link (1816) as Cladosporium herbarum (Pers.: Fr.). Cladosporium is currently only known as the asexual morph, which is characterised by erect, straight or geniculate conidiophores, abundant branched acropetal chains of smooth to roughened dry conidia produced from mono- or polyblastic conidiogenous cells, the coronate structure of conidiogenous loci and conidial hila, consisting of a central convex dome surrounded by a raised periclinal rim (David 1997, Crous et al. 2007).

To clarify the relationship of species in the complex Cladosporium, subsequent researchers have been constantly revising this genus and the use of molecular analysis is necessary as well as morphological characters (David 1997, Dugan et al. 2004, Heuchert et al. 2005, Schubert 2005, Schubert et al. 2007, Crous et al. 2007, Sandoval-Denis et al. 2016, Bezerra et al. 2017, Bezerra et al. 2017, Abdollahzadeh et al. 2020). Some phylogenetic studies have proposed a multi-locus sequence analysis approach to clarify species diversity within the genus with internal spacers of the rDNA genes (ITS), translation elongation factor 1- $\alpha$ (TEF1- $\alpha$ ) and actin (ACT) (Bensch et al. 2012, Bensch et al. 2015, Bensch et al. 2018, Tibpromma et al. 2019, Iturrieta-González et al. 2021, Zimowska et al. 2021). Based on the phylogenetic analyses and morphological features, about 237 species have been accepted within the genus, which are split into three species complexes, Cladosporium herbaum (Schubert et al. 2007), C. sphaerospermum (Zalar et al. 2007, Dugan et al. 2008) and C. cladosporioides (Bensch et al. 2010).

The species of Cladosporium are able to colonise a wide range of substrates and can be isolated in any natural or anthropogenically-affected environment (Flannigan et al. 2002, Bensch et al. 2010, Bensch et al. 2012, Bensch et al. 2018, Sandoval-Denis et al. 2015, Temperini et al. 2018, Chung et al. 2019). They are well known as plant pathogens, which may occur on leaves, stems and fruits on different plants, for example, Asparagaceae, Asteraceae, Fabaceae, Myrtaceae, Orchidaceae and Poaceae (Schubert 2005, Bensch et al. 2012, Bensch et al. 2015, Marin-Felix et al. 2017, Rosado et al. 2019). Besides, some species have been reported as pathogens of animals and humans, saprobes and endophytes and been also reported as hyperparasites on other fungi (Sandoval-Denis et al. 2015, Sandoval-Denis et al. 2016, Zhou et al. 2016, Velázquez-Jiménez et al. 2019). Furthermore, some species have shown the ability to produce medicinal compounds or their potential as biological agents to control plant diseases (Köhl et al. 2015, Khan et al. 2016, Adorisio et al. 2019). 
During the investigation of pathogenic fungi on leaf spots of medicinal plants in Yunnan Province, China, two new species Cladosporium yunnanensis and C. paris were identified, based on morphology and multi-gene phylogenetic analysis. Full descriptions, illustrations and update of the phylogenetic backbone tree for Cladosporium are provided as well.

\section{Materials and methods}

\section{Isolation and morphological examination}

Leaf specimens with disease symptoms of cultivated Paris polyphylla were collected from Dali, Yunnan Province, China in October and November 2020 and taken back to the laboratory in an envelope. The leaves were kept at $4^{\circ} \mathrm{C}$ in Zip-lock plastic bags before they were processed in the laboratory. Single spore isolations were made onto potato dextrose agar (PDA). After 8-10 hours, a single germinating conidia was transferred aseptically to a new PDA plate to obtain cultures and grow at $20-25^{\circ} \mathrm{C}$ in daylight (Chomnunti et al. 2014).

The cultures are deposited in Kunming Institute of Botany, Chinese Academy of Sciences (KUNCC) and China General Microbiological Culture Collection Center (CGMCC). Cultures are deposited at the Herbarium of Cryptogams Kunming Institute of Botany Academia Sinica (Herb. HKAS). Facesoffungi and Index Fungorum numbers were obtained as in Jayasiri et al. (2015) and Index Fungorum.

\section{DNA extraction, PCR amplification and sequencing}

Genomic DNA was extracted from fresh mycelium grown on PDA at room temperature $\left(25^{\circ} \mathrm{C}\right)$. The Trelief ${ }^{\mathrm{TM}}$ Plant Genomic DNA Kit (TSP101) was used to extract DNA according to the manufacturer's instructions. ITS, TEF1- $\alpha$ and ACT gene regions were amplified using the primer pairs ITS1/ITS4, EF1-728F/EF1-986R and ACT-512F/ACT-783R. The final volume of the PCR reaction was $25 \mu \mathrm{l}$ and contained $12.5 \mu \mathrm{l}$ of $2 \times$ Power Taq PCR MasterMix (a premix and ready to use solution, including 0.1 Units/ $\mu$ Taq DNA Polymerase, $500 \mu \mathrm{M}$ dNTP Mixture each (dATP, dCTP, dGTP, dTTP), $20 \mathrm{mM}$ Tris- $\mathrm{HCl} \mathrm{pH}$ $8.3,100 \mathrm{mM} \mathrm{KCl}, 3 \mathrm{mM} \mathrm{MgCl} 2$, stabiliser and enhancer), $1 \mu \mathrm{l}$ of each primer $(10 \mu \mathrm{M}), 1 \mu \mathrm{l}$ genomic DNA extract and $9.5 \mu \mathrm{l}$ deionised water. The PCR thermal cycle programme for ITS, TEF1- $\alpha$ and ACT amplification was as follows: initial denaturation of $94^{\circ} \mathrm{C}$ for 3 minutes, followed by 35 cycles of denaturation at $94^{\circ} \mathrm{C}$ for 45 seconds, annealing at $56^{\circ} \mathrm{C}$ for 50 seconds, elongation at $72^{\circ} \mathrm{C}$ for 1 minute and the final extension at $72^{\circ} \mathrm{C}$ for 10 minutes. PCR products were purified using minicolumns, purification resin and buffer according to the manufacturer's protocols (Amershamproduct code: 27-9602-01). The sequencing works were carried by Tsingke Biological Engineering Technology and Services Co., Ltd (Yunnan, P.R. China).

\section{Phylogenetic analysis}

Sequence data for relevant strains were downloaded from GenBank following latest publications (Freitas 2018, Iturrieta-González et al. 2021, Zimowska et al. 2021). The sequences aligned using MAFFTv.7 (http://mafft.cbrc.jp/alignment/server/) (Katoh and 
Standley 2013) and optimised manually when needed. The aligned dataset was analysed by Maximum Likelihood (ML) and Bayesian Inference $(\mathrm{BI})$.

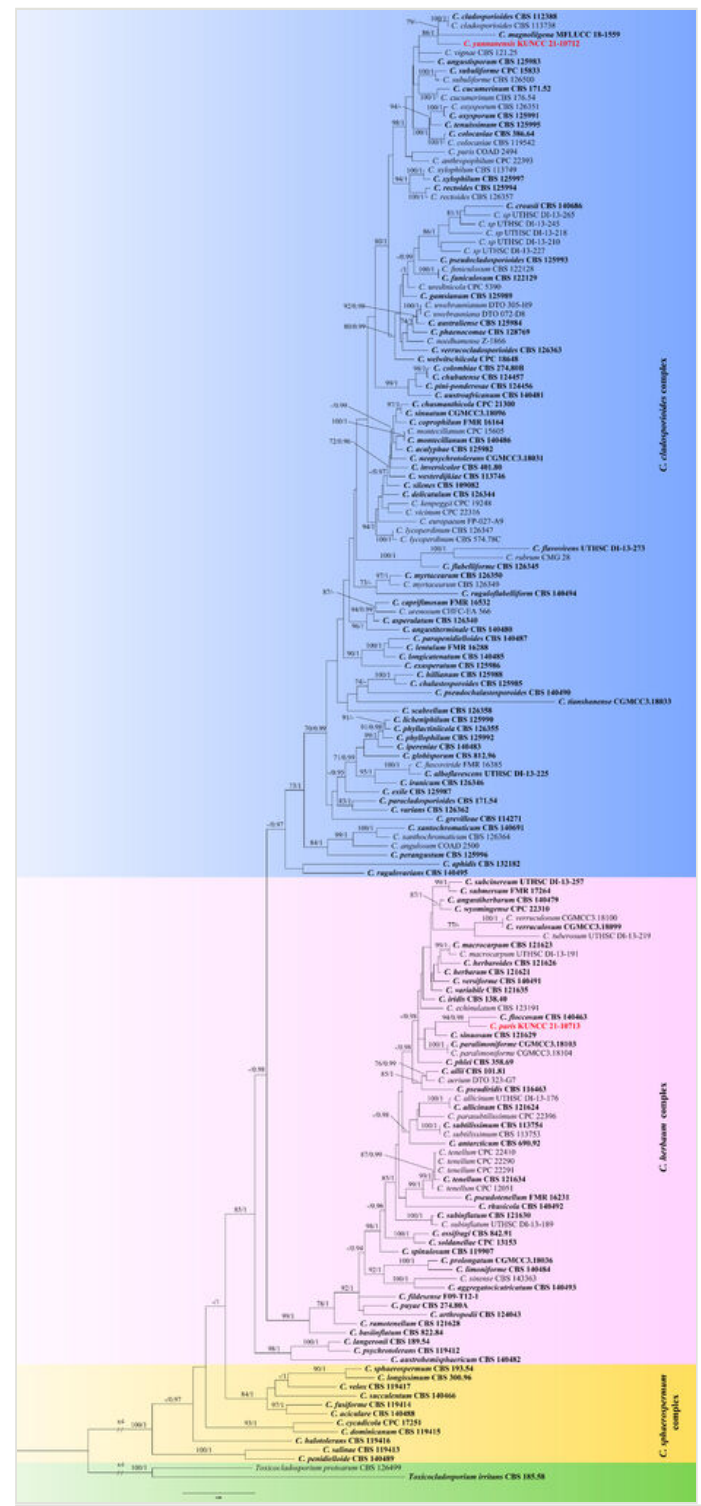

Figure 1. doi

Maximum Likelihood (ML) tree obtained from the combined analysis of ITS, TEF1- $\alpha$ and ACT sequences of 161 strains from Cladosporium. The tree is rooted with Toxicocladosporium irritans (CBS 185.58) and T. protearum (CBS 126499). Numbers on the branches represent ML bootstrap support values (MLBS) $\geq 70 \%$, followed by Bayesian posterior probabilities (PP) $\geq 0.95$, lower values are indicated as "-". Names of species newly described are indicated in red and ex-type strains and reference specimens are indicated in bold. Branch lengths are proportional to distance. 
Maximum Likelihood analysis was performed by using RAxMLGUI v.1.3 (Silvestro and Michalak 2012). The optimal ML tree search was conducted with 1,000 separate runs using the default algorithm of the programme from a random starting tree for each run. The final tree was selected amongst suboptimal trees from each run by comparing the likelihood scores using the GTR+GAMMA substitution model. Maximum Likelihood bootstrap values equal to or greater than $70 \%$ were given as the first set of numbers above the nodes in the resulting ML tree (Fig. 1).

Bayesian analysis was conducted with MrBayes v.3.1.2 (Ronquist and Huelsenbeck 2003) to evaluate posterior probabilities (Rannala and Yang 1996) by Markov Chain Monte Carlo sampling (MCMC). The best-fit models of evolution were estimated by MrModeltest v.2.2 (Nylander 2004). ITS selected the SYM+I+G model with inverse gamma-distributed rate in Bayesian analyses. TEF1- $\alpha$ and ACT selected the GTR+I+G model with inverse gammadistributed rate in Bayesian analyses. The robustness of $\mathrm{ML}$ analyses was evaluated by bootstrap support (MLBS). The parameter settings, used in these analyses, were two simultaneous runs of $10,000,000$ generations and four Markov chains, sampled every 1,000 generations. The $50 \%$ majority rule consensus tree and posterior probability values (PP) were calculated after discarding the first $25 \%$ of the samples. A PP value of $\geq 0.95$ was considered significant (Hespanhol et al. 2019).

The phylogenetic trees were viewed and optimised in FigTree v.1.2.2 (Rambaut and Drummond 2008) and edited further using Microsoft Office PowerPoint. Newly-generated sequences in this study were deposited in GenBank (Table 1).

Table 1.

Isolates and sequences used in this study (newly-generated sequences are indicated with a "*”, strains isolated from the holotype and reference specimens are indicated in bold).

\begin{tabular}{|c|c|c|c|c|}
\hline \multirow[t]{2}{*}{ Species } & \multirow[t]{2}{*}{ Strain number } & \multicolumn{3}{|c|}{ GenBank Accession Numbers } \\
\hline & & ITS & TEF1- $\alpha$ & ACT \\
\hline Cladosporium acalyphae & CBS 125982 & NR_119835 & HM148235 & HM148481 \\
\hline C. aciculare & CBS 140488 & KT600411 & KT600509 & KT600607 \\
\hline C. aerium & DTO 323-G7 & MF472899 & MF473326 & MF473749 \\
\hline C. aggregatocicatricatum & CBS 140493 & $\underline{\text { NR_152300 }}$ & $\underline{\text { KT600547 }}$ & $\underline{\mathrm{KT} 600645}$ \\
\hline C. alboflavescens & UTHSC DI-13-225 & LN834420 & LN834516 & LN834604 \\
\hline C. allicinum & CBS 121624 & NR_152266 & EF679425 & EF679502 \\
\hline C. allicinum & UTHSC DI-13-176 & LN834354 & LN834450 & LN834538 \\
\hline C. allii & CBS 101.81 & JN906977 & JN906983 & JN906996 \\
\hline C. angulosum & COAD 2500 & MK253346 & MK293786 & MK249989 \\
\hline C. angustiherbarum & CBS 140479 & NR_152286 & KT600475 & KT600574 \\
\hline C. angustisporum & CBS 125983 & NR_111530 & HM148236 & HM148482 \\
\hline C. angustiterminale & CBS 140480 & NR_152287 & KT600476 & KT600575 \\
\hline
\end{tabular}




\begin{tabular}{|c|c|c|c|c|}
\hline \multirow[t]{2}{*}{ Species } & \multirow[t]{2}{*}{ Strain number } & \multicolumn{3}{|c|}{ GenBank Accession Numbers } \\
\hline & & ITS & TEF1- $\alpha$ & ACT \\
\hline C. antarcticum & CBS 690.92 & NR_121332 & EF679405 & EF679484 \\
\hline C. anthropophilum & CPC 22393 & MF472922 & MF473349 & MF473772 \\
\hline C. aphidis & CBS 132182 & $\underline{\mathrm{JN} 906978}$ & JN906984 & JN906997 \\
\hline C. arenosum & CHFC-EA 566 & MN879328 & MN890011 & MN890008 \\
\hline C. arthropodii & CBS 124043 & NR_120011 & $\underline{\mathrm{JN} 906985}$ & JN906998 \\
\hline C. asperulatum & CBS 126340 & NR_119836 & HM148239 & HM148485 \\
\hline C. australiense & CBS 125984 & NR_119837 & $\underline{\text { HM148240 }}$ & $\underline{\text { HM148486 }}$ \\
\hline C. austroafricanum & CBS 140481 & NR_152288 & $\underline{\text { KT600478 }}$ & KT600577 \\
\hline C. austrohemisphaericum & CBS 140482 & KT600382 & $\underline{\text { KT600479 }}$ & $\underline{\mathrm{KT} 600578}$ \\
\hline C. basiinflatum & CBS 822.84 & NR_111531 & HM148241 & $\underline{\text { HM148487 }}$ \\
\hline C. caprifimosum & FMR 16532 & $\underline{\text { LR813198 }}$ & $\underline{\text { LR813210 }}$ & $\underline{\text { LR813205 }}$ \\
\hline C. chalastosporoides & CBS 125985 & NR_119838 & $\underline{\text { HM148242 }}$ & $\underline{\text { HM148488 }}$ \\
\hline C. chasmanthicola & CPC 21300 & NR_152307 & $\underline{\text { KY646227 }}$ & $\underline{\text { KY646224 }}$ \\
\hline C. chubutense & CBS 124457 & NR_119728 & FJ936161 & FJ936165 \\
\hline C. cladosporioides & CBS 112388 & NR_119839 & $\underline{\text { HM148244 }}$ & $\underline{\text { HM148490 }}$ \\
\hline C. cladosporioides & CBS 113738 & $\underline{\mathrm{HM} 148004}$ & $\underline{\text { HM148245 }}$ & HM148491 \\
\hline C. colocasiae & CBS 386.64 & NR_119840 & $\underline{\text { HM148310 }}$ & $\underline{\text { HM148555 }}$ \\
\hline C. colocasiae & CBS 119542 & $\underline{\mathrm{HM} 148066}$ & HM148309 & $\underline{\text { HM148554 }}$ \\
\hline C. colombiae & CBS 274.80B & NR_119729 & FJ936163 & $\underline{\text { FJ936166 }}$ \\
\hline C. coprophilum & FMR 16164 & LR813201 & LR813213 & $\underline{\text { LR813207 }}$ \\
\hline C. crousii & CBS 140686 & LN834431 & LN834527 & $\underline{\text { LN834615 }}$ \\
\hline C. cucumerinum & CBS 171.52 & NR_119841 & $\underline{\text { HM148316 }}$ & $\underline{\text { HM148561 }}$ \\
\hline C. cucumerinum & CBS 176.54 & $\underline{\text { HM148078 }}$ & $\underline{\text { HM148322 }}$ & $\underline{\mathrm{HM} 148567}$ \\
\hline C. cycadicola & CPC 17251 & KJ869122 & KJ869236 & KJ869227 \\
\hline C. delicatulum & CBS 126344 & MH863920 & HM148325 & HM148570 \\
\hline C. dominicanum & CBS 119415 & DQ780353 & JN906986 & EF101368 \\
\hline C. echinulatum & CBS 123191 & JN906980 & JN906987 & JN906999 \\
\hline C. europaeum & FP-027-A9 & $\underline{\mathrm{MH} 102078}$ & $\underline{\mathrm{MH} 102121}$ & MH102068 \\
\hline C. exasperatum & CBS 125986 & NR_119843 & HM148334 & $\underline{\text { HM148579 }}$ \\
\hline C. exile & CBS 125987 & NR_111532 & $\underline{\text { HM148335 }}$ & $\underline{\text { HM1 } 148580}$ \\
\hline C. fildesense & F09-T12-1 & JX845290 & MN233633 & MN233632 \\
\hline C. flabelliforme & CBS 126345 & NR_119844 & $\underline{\text { HM148336 }}$ & $\underline{\text { HM148581 }}$ \\
\hline C. flavovirens & UTHSC DI-13-273 & LN834440 & $\underline{\text { LN834536 }}$ & LN834624 \\
\hline C. floccosum & CBS 140463 & $\underline{\mathrm{LN} 834416}$ & $\underline{\text { LN834512 }}$ & $\underline{\text { LN834600 }}$ \\
\hline C. funiculosum & CBS 122129 & NR_119845 & HM148338 & HM148583 \\
\hline
\end{tabular}




\begin{tabular}{|c|c|c|c|c|}
\hline \multirow[t]{2}{*}{ Species } & \multirow[t]{2}{*}{ Strain number } & \multicolumn{3}{|c|}{ GenBank Accession Numbers } \\
\hline & & ITS & TEF1- $\alpha$ & ACT \\
\hline C. funiculosum & CBS 122128 & $\underline{\mathrm{HM} 148093}$ & HM148337 & $\underline{\mathrm{HM} 148582}$ \\
\hline C. fuscoviride & FMR 16385 & $\underline{\text { LR813200 }}$ & $\underline{\text { LR813212 }}$ & $\underline{\text { LR813206 }}$ \\
\hline C. fusiforme & CBS 119414 & DQ780388 & JN906988 & EF101372 \\
\hline C. gamsianum & CBS 125989 & NR_111533 & HM148339 & HM148584 \\
\hline C. globisporum & CBS 812.96 & NR_111534 & $\underline{\text { HM148340 }}$ & HM148585 \\
\hline C. grevilleae & CBS 114271 & NR_119960 & JF770472 & $\underline{\mathrm{JF} 770473}$ \\
\hline C. halotolerans & CBS 119416 & DQ780364 & JN906989 & EF101397 \\
\hline C. herbaroides & CBS 121626 & NR_119655 & EF679432 & EF679509 \\
\hline C. herbarum & CBS 121621 & NR_119656 & EF679440 & EF679516 \\
\hline C. hillianum & CBS 125988 & $\underline{\text { NR_119846 }}$ & $\underline{\text { HM148341 }}$ & $\underline{\text { HM148586 }}$ \\
\hline C. inversicolor & CBS 401.80 & NR_111535 & $\underline{\text { HM148345 }}$ & HM148590 \\
\hline C. ipereniae & CBS 140483 & NR_152290 & KT600491 & $\underline{\text { KT600589 }}$ \\
\hline C. iranicum & CBS 126346 & $\underline{\text { NR_111536 }}$ & $\underline{\text { HM148354 }}$ & $\underline{\text { HM148599 }}$ \\
\hline C. iridis & CBS 138.40 & NR_111271 & EF679447 & EF679523 \\
\hline C. kenpeggii & CPC 19248 & KY646222 & KY646228 & KY646225 \\
\hline C. langeronii & CBS 189.54 & DQ780379 & JN906990 & EF101357 \\
\hline C. lentulum & FMR 16288 & LR813203 & $\underline{\text { LR813215 }}$ & LR813209 \\
\hline C. licheniphilum & CBS 125990 & NR_119847 & HM148355 & HM148600 \\
\hline C. limoniforme & CBS 140484 & KT600397 & KT600494 & KT600592 \\
\hline C. longicatenatum & CBS 140485 & NR_152291 & $\underline{\text { KT600500 }}$ & $\underline{\text { KT600598 }}$ \\
\hline C. longissimum & CBS 300.96 & DQ780352 & EU570259 & EF101385 \\
\hline C. lycoperdinum & CBS 126347 & MH863923 & $\underline{\mathrm{HM} 148356}$ & $\underline{\mathrm{HM} 148601}$ \\
\hline C. Iycoperdinum & CBS $574.78 \mathrm{C}$ & $\underline{\text { HM148115 }}$ & $\underline{\text { HM148359 }}$ & $\underline{\mathrm{HM} 148604}$ \\
\hline C. macrocarpum & CBS 121623 & NR_119657 & EF679453 & EF679529 \\
\hline C. macrocarpum & UTHSC DI-13-191 & $\underline{\text { LN834379 }}$ & $\underline{\text { LN834475 }}$ & LN834563 \\
\hline C. magnoliigena & MFLUCC 18-1559 & MK347813 & MK340864 & - \\
\hline C. montecillanum & CBS 140486 & NR 152292 & $\underline{\text { KT600504 }}$ & KT600602 \\
\hline C. montecillanum & CPC 15605 & $\underline{\mathrm{KT} 600407}$ & $\underline{\mathrm{KT} 600505}$ & $\underline{\text { KT600603 }}$ \\
\hline C. myrtacearum & CBS 126349 & MH863925 & $\underline{\mathrm{HM} 148360}$ & $\underline{\mathrm{HM} 148605}$ \\
\hline C. myrtacearum & CBS 126350 & NR_119849 & HM148361 & $\underline{H M 148606}$ \\
\hline C. needhamense & Z-1866 & MF473142 & MF473570 & MF473991 \\
\hline C. neopsychrotolerans & CGMCC3.18031 & KX938383 & KX938400 & KX938366 \\
\hline C. ossifragi & CBS 842.91 & NR_121333 & EF679459 & EF679535 \\
\hline C. oxysporum & CBS 125991 & NR_152267 & $\underline{\text { HM148362 }}$ & $\underline{\mathrm{HM} 148607}$ \\
\hline C. oxysporum & CBS 126351 & MH863927 & $\underline{\mathrm{HM} 148363}$ & $\underline{\mathrm{HM} 148608}$ \\
\hline
\end{tabular}




\begin{tabular}{|c|c|c|c|c|}
\hline \multirow[t]{2}{*}{ Species } & \multirow[t]{2}{*}{ Strain number } & \multicolumn{3}{|c|}{ GenBank Accession Numbers } \\
\hline & & ITS & TEF1- $\alpha$ & ACT \\
\hline C. paracladosporioides & CBS 171.54 & NR_119850 & HM148364 & HM148609 \\
\hline C. paralimoniforme & CGMCC3.18103 & $\underline{\mathrm{KX938392}}$ & $\underline{\mathrm{KX938409}}$ & $\underline{\mathrm{KX} 938375}$ \\
\hline C. paralimoniforme & CGMCC3.18104 & $\underline{\mathrm{KX} 938393}$ & $\underline{\mathrm{KX} 938410}$ & $\underline{\mathrm{KX} 938376}$ \\
\hline C. parapenidielloides & CBS 140487 & NR_152293 & KT600508 & KT600606 \\
\hline C. parasubtilissimum & CPC 22396 & MF473171 & MF473594 & MF474019 \\
\hline C. paris sp. nov. ${ }^{*}$ & KUN HKAS $121701^{*}$ & OK338503* & $\underline{\text { OL825681* }}^{*}$ & $\underline{\text { OL466938* }}$ \\
\hline C. penidielloide & CBS 140489 & $\underline{\text { KT600412 }}$ & $\underline{\text { KT600510 }}$ & $\underline{\text { KT600608 }}$ \\
\hline C. perangustum & CBS 125996 & NR_119851 & HM148365 & $\underline{\text { HM148610 }}$ \\
\hline C. phaenocomae & CBS 128769 & NR_119950 & $\underline{\text { JF499875 }}$ & $\underline{\text { JF499881 }}$ \\
\hline C. phlei & CBS 358.69 & NR_120013 & JN906991 & JN907000 \\
\hline C. phyllactiniicola & CBS 126355 & NR_111537 & $\underline{\text { HM148397 }}$ & HM148642 \\
\hline C. phyllophilum & CBS 125992 & NR_111538 & HM148398 & HM148643 \\
\hline C. pini-ponderosae & CBS 124456 & $\underline{\text { NR_119730 }}$ & FJ936164 & FJ936167 \\
\hline C. prolongatum & CGMCC3.18036 & $\underline{\text { KX938394 }}$ & $\underline{\mathrm{KX} 938411}$ & $\underline{\mathrm{KX} 938377}$ \\
\hline C. pseudiridis & CBS 116463 & NR_111272 & EF679461 & EF679537 \\
\hline C. pseudochalastosporoides & CBS 140490 & NR_152296 & $\underline{\mathrm{KT} 600513}$ & $\underline{\mathrm{KT} 600611}$ \\
\hline C. pseudocladosporioides & CBS 125993 & $\underline{\text { NR_119852 }}$ & $\underline{\text { HM148402 }}$ & HM148647 \\
\hline C. pseudotenellum & FMR 16231 & LR813145 & $\underline{\text { LR813196 }}$ & $\underline{\text { LR813146 }}$ \\
\hline C. psychrotolerans & CBS 119412 & DQ780386 & JN906992 & EF101365 \\
\hline C. puris & COAD 2494 & MK253338 & MK293778 & MK249981 \\
\hline C. puyae & CBS 274.80A & NR_152298 & $\underline{\text { KT600516 }}$ & $\underline{\mathrm{KT} 600614}$ \\
\hline C. ramotenellum & CBS 121628 & NR_119658 & EF679462 & EF679538 \\
\hline C. rectoides & CBS 125994 & NR_111539 & $\underline{\text { HM148438 }}$ & HM148683 \\
\hline C. rectoides & CBS 126357 & $\underline{\mathrm{MH} 863933}$ & $\underline{\text { HM148439 }}$ & $\underline{\text { HM148684 }}$ \\
\hline C. rhusicola & CBS 140492 & NR_152299 & KT600539 & KT600637 \\
\hline C. rubrum & CMG 28 & $\underline{\text { MN053018 }}$ & $\underline{\text { MN066644 }}$ & MN066639 \\
\hline C. ruguloflabelliform & CBS 140494 & $\underline{\text { KT600458 }}$ & KT600557 & $\underline{\mathrm{KT} 600655}$ \\
\hline C. rugulovarians & CBS 140495 & $\underline{\mathrm{KT} 600459}$ & KT600558 & $\underline{\mathrm{KT} 600656}$ \\
\hline C. salinae & CBS 119413 & DQ780374 & JN906993 & EF101390 \\
\hline C. scabrellum & CBS 126358 & NR_119853 & $\underline{\text { HM148440 }}$ & $\underline{\text { HM148685 }}$ \\
\hline C. silenes & CBS 109082 & $\underline{\text { NR_111270 }}$ & EF679429 & EF679506 \\
\hline C. sinense & CBS 143363 & $\underline{\text { MF473252 }}$ & $\underline{\text { MF473675 }}$ & MF474102 \\
\hline C. sinuatum & CGMCC3.18096 & $\underline{K X 938385}$ & KX938402 & $\underline{K X 938368}$ \\
\hline C. sinuosum & CBS 121629 & NR_119659 & EF679464 & EF679540 \\
\hline C. soldanellae & CPC 13153 & NR_120014 & JN906994 & JN907001 \\
\hline
\end{tabular}




\begin{tabular}{|c|c|c|c|c|}
\hline \multirow[t]{2}{*}{ Species } & \multirow[t]{2}{*}{ Strain number } & \multicolumn{3}{|c|}{ GenBank Accession Numbers } \\
\hline & & ITS & TEF1- $\alpha$ & ACT \\
\hline C. sp. & UTHSC DI-13-227 & $\underline{\text { LN834422 }}$ & $\underline{L} 8 \mathrm{~N} 34518$ & $\underline{\text { LN834606 }}$ \\
\hline C. sp. & UTHSC DI-13-245 & $\underline{\text { LN834429 }}$ & $\underline{\text { LN834525 }}$ & $\underline{\mathrm{LN} 834613}$ \\
\hline C. sp. & UTHSC DI-13-265 & $\underline{\text { LN834435 }}$ & $\underline{\text { LN834531 }}$ & $\underline{\text { LN834619 }}$ \\
\hline C. sp. & UTHSC DI-13-218 & $\underline{\mathrm{LN} 834418}$ & $\underline{\text { LN834514 }}$ & LN834602 \\
\hline C. sp. & UTHSC DI-13-210 & $\underline{\mathrm{LN} 834414}$ & $\underline{\text { LN834510 }}$ & $\underline{\text { LN834598 }}$ \\
\hline C. sphaerospermum & CBS 193.54 & $\underline{\text { NR_111222 }}$ & EU570261 & EU570269 \\
\hline C. spinulosum & CBS 119907 & NR_119660 & EF679466 & EF679542 \\
\hline C. subcinereum & UTHSC DI-13-257 & NR_148193 & LN834529 & LN834617 \\
\hline C. subinflatum & UTHSC DI-13-189 & $\underline{\text { LN834391 }}$ & $\underline{\text { LN834487 }}$ & $\underline{\text { LN834575 }}$ \\
\hline C. subinflatum & CBS 121630 & $\underline{\text { NR_119661 }}$ & EF679467 & EF679543 \\
\hline C. submersum & FMR 17264 & $\underline{\text { LR813144 }}$ & $\underline{\text { LR813197 }}$ & $\underline{\text { LR813195 }}$ \\
\hline C. subtilissimum & CBS 113754 & $\underline{\text { NR } 111273}$ & EF679475 & EF679551 \\
\hline C. subtilissimum & CBS 113753 & EF679396 & EF679474 & EF679550 \\
\hline C. subuliforme & CBS 126500 & NR_119854 & $\underline{\text { HM148441 }}$ & $\underline{\text { HM148686 }}$ \\
\hline C. subuliforme & CPC 15833 & $\underline{\text { KT600453 }}$ & KT600552 & $\underline{\text { KT600650 }}$ \\
\hline C. succulentum & CBS 140466 & LN834434 & LN834530 & $\underline{\text { LN834618 }}$ \\
\hline C. tenellum & CBS 121634 & NR_119662 & EF679479 & EF679555 \\
\hline C. tenellum & CPC 22410 & MF473280 & MF473703 & MF474130 \\
\hline C. tenellum & CPC 12051 & EF679400 & EF679478 & EF679554 \\
\hline C. tenellum & CPC 22291 & MF473279 & MF473702 & MF474129 \\
\hline C. tenellum & CPC 22290 & $\underline{\text { MF473278 }}$ & MF473701 & $\underline{\text { MF474128 }}$ \\
\hline C. tenuissimum & CBS 125995 & $\underline{\text { NR_119855 }}$ & $\underline{\text { HM148442 }}$ & $\underline{\text { HM148687 }}$ \\
\hline C. tianshanense & CGMCC3.18033 & $\underline{\mathrm{KX} 938381}$ & $\underline{\mathrm{KX} 938398}$ & $\underline{\mathrm{KX} 938364}$ \\
\hline C. tuberosum & UTHSC DI-13-219 & $\underline{\mathrm{LN} 834419}$ & $\underline{\text { LN834515 }}$ & $\underline{\text { LN834603 }}$ \\
\hline C. uredinicola & CPC 5390 & $\underline{\mathrm{AY} 251071}$ & $\underline{\text { HM148467 }}$ & $\underline{\mathrm{HM} 148712}$ \\
\hline C. uwebrauniana & DTO 072-D8 & MF473306 & MF473729 & MF474156 \\
\hline C. uwebraunianum & DTO 305-H9 & $\underline{\text { MF473307 }}$ & MF473730 & $\underline{\text { MF474157 }}$ \\
\hline C. variabile & CBS 121635 & $\underline{\text { NR_119663 }}$ & EF679481 & EF679557 \\
\hline C. varians & CBS 126362 & $\underline{\text { NR } \_119856}$ & $\underline{\mathrm{HM} 148470}$ & $\underline{\text { HM148715 }}$ \\
\hline C. velox & CBS 119417 & $\underline{D Q 780361}$ & $\underline{\mathrm{JN} 906995}$ & EF101388 \\
\hline C. verrucocladosporioides & CBS 126363 & $\underline{\text { NR_111540 }}$ & $\underline{\text { HM148472 }}$ & HM148717 \\
\hline C. verruculosum & CGMCC3.18099 & KX938388 & KX938405 & KX938371 \\
\hline C. verruculosum & CGMCC3.18100 & $\underline{K X 938389}$ & $\underline{\mathrm{KX} 938406}$ & $\underline{\mathrm{KX} 938372}$ \\
\hline C. versiforme & CBS 140491 & NR_152297 & $\underline{\text { KT600515 }}$ & $\underline{\mathrm{KT} 600613}$ \\
\hline C. vicinum & CPC 22316 & $\underline{\text { MF473311 }}$ & MF473734 & MF474161 \\
\hline
\end{tabular}




\begin{tabular}{|c|c|c|c|c|}
\hline \multirow[t]{2}{*}{ Species } & \multirow[t]{2}{*}{ Strain number } & \multicolumn{3}{|c|}{ GenBank Accession Numbers } \\
\hline & & ITS & TEF1- $\alpha$ & ACT \\
\hline C. vignae & CBS 121.25 & $\underline{\mathrm{HM} 148227}$ & HM148473 & $\underline{\mathrm{HM} 148718}$ \\
\hline C. welwitschiicola & CPC 18648 & NR 152308 & KY646229 & KY646226 \\
\hline C. westerdijkiae & CBS 113746 & HM148061 & HM148303 & HM148548 \\
\hline C. wyomingense & CPC 22310 & MF473315 & MF473738 & MF474165 \\
\hline C. xanthochromaticum & CBS 126364 & $\underline{\text { HM148122 }}$ & $\underline{\mathrm{HM} 148366}$ & $\underline{\mathrm{HM} 148611}$ \\
\hline C. xantochromaticum & CBS 140691 & LN834415 & LN834511 & LN834599 \\
\hline C. xylophilum & CBS 125997 & NR_111541 & HM148476 & HM148721 \\
\hline C. xylophilum & CBS 113749 & HM148228 & HM148474 & $\underline{\text { HM} 148719}$ \\
\hline C. yunnanensis sp. nov. ${ }^{*}$ & KUN HKAS 121704* & OK338502* & OL825680* & OL466937* \\
\hline Toxicocladosporium irritans & CBS 185.58 & NR_152316 & - & LTT821375 \\
\hline Toxicocladosporium protearum & CBS 126499 & NR_152321 & - & LT821379 \\
\hline
\end{tabular}

\section{Taxon treatments}

\section{Cladosporium yunnanensis H.W. Shen, Y.X. Xu, H.Y. Su \& Z.L. Luo, sp. nov.}

- IndexFungorum 558843

- $\quad$ Species-ID Facesoffungi number: FoF 10538

\section{Material}

Holotype:

a. scientificName: Cladosporium yunnanensis; kingdom: Fungi; phylum: Ascomycota; class: Dothideomycetes; order: Capnodiales; family: Cladosporiaceae; genus: Cladosporium; locationRemarks: China, Yunnan Province, Dali, on diseased leaves of Paris polyphylla, 2 October 2020; day: 2020; habitat: leaf spots of Paris polyphylla; recordedBy: Yue-Xin Xu; collectionID: 1CL JD 5-1-4; collectionCode: Y-23

\section{Description}

Asexual morph: hyphomycetous (Fig. 2). Mycelium superficial and immersed, composed of septate, branched, subhyaline, smooth-walled, 1-3 $\mu \mathrm{m}$ wide. Conidiophores macronematous, $127-190 \times 4-6 \mu \mathrm{m}(\overline{\mathrm{x}}=158.2 \times 5.1 \mu \mathrm{m}, \mathrm{n}=15)$, solitary or in small loose groups, erect to slightly flexuous, non-nodulose, sometimes subnodulose at the uppermost apex, unbranched, 0-6 septate, sometimes slightly constricted at septa, pale brown, smooth, sometimes somewhat irregularly roughwalled or verruculose. Conidiogenous cells terminal and intercalary, loci crowded at the apex forming clusters of pronounced scars, 1-2 conidiogenous loci formed at about the same level, loci often situated at lateral shoulders due to sympodial proliferation, loci 1$2 \mu \mathrm{m}$ diam. Conidia solitary or in short unbranched chains, straight to slightly curved, cylindrical-oblong, $7-19 \times 5-7 \mu \mathrm{m}(\overline{\mathrm{x}}=13.2 \times 5.7 \mu \mathrm{m}, \mathrm{n}=30), 0-3$ septate, sometimes 
slightly constricted at the septa, pale to pale medium olivaceous-brown. Sexual morph: Undetermined.

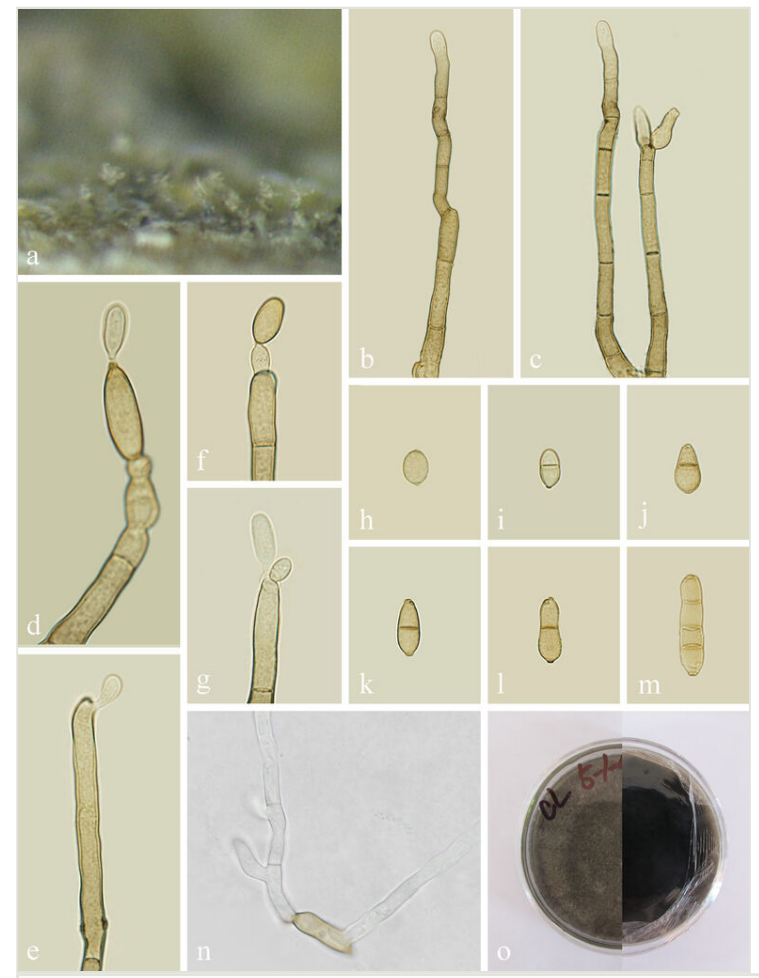

Figure 2. doi

Cladosporium yunnanensis (KUN-HKAS 121704, holotype). a Colonies; b-c Conidiophores; d-g Conidiogenous cells with conidia; $\mathbf{h}-\mathbf{m}$ Conidia; $\mathbf{n}$ Germinating conidium; o Culture on PDA from above and reverse. Scale bars: $\mathbf{b}-\mathbf{d}=20 \mu \mathrm{m} ; \mathbf{e}-\mathbf{k}=15 \mu \mathrm{m} ; \mathbf{I}=30 \mu \mathrm{m}$.

Culture characteristics: Colonies on PDA attaining $25 \mathrm{~mm}$ diam. after $7 \mathrm{~d}, 45 \mathrm{~mm}$ diam. after $14 \mathrm{~d}$ and covering the whole Petridish after $30 \mathrm{~d}$, dark green to olive green, velvety, furrowed; reverse dark green to black.

Material examined: China, Yunnan Province, Dali, on diseased leaves of Paris polyphylla, 2 October 2020, Y.X. Xu, Y-23. (KUN-HKAS 121704, holotype), ex-type living culture CGMCC $3.20622=$ KUNCC 21-10712

\section{Etymology}

"yunnanensis" refers to Yunnan Province, China, where the species was collected.

\section{Distribution}

China, Yunnan Province, Dali, on diseased leaves of Paris polyphylla 


\section{Notes}

Based on the multi-locus phylogenetic analysis (Fig. 1), Cladosporium yunnanensis grouped in a well-supported clade, together with $C$. cladosporioides and $C$. magnoliigena. However, the genetic distance allows it to be considered a distinct species within the clade (Fig. 1). Morphologically, C. yunnanensis has much shorter conidiophores than C. cladosporioides (up to $190 \mu \mathrm{m}$ vs. up to $350 \mu \mathrm{m}$ ), but longer than C. magnoliigena (up to $190 \mu \mathrm{m}$ vs. up to $150 \mu \mathrm{m}$ ). Moreover, the new species differs from $C$. cladosporioides by the smaller conidiogenous cells $(7-19 \times 5-7 \mu \mathrm{m}$ vs. 4-18 $\times 2-5 \mu \mathrm{m})$, but larger than C. magnoliigena $(7-19 \times 5-7 \mu \mathrm{m}$ vs. $4-18 \times 2-5 \mu \mathrm{m})$ (Bensch et al. 2012, Jayasiri et al. 2019). The BLAST analysis of TEF1- $\alpha$ and ACT shows that $C$. yunnanensis (KUN-HKAS 121704) is different from C. cladosporioides (CBS 112388) by 16 and 10 nucleotide differences, respectively and the comparison of TEF1- $\alpha$ between C. yunnanensis (KUN-HKAS 121704) and C. magnoligena (CBS 140463) reveals 33 nucleotide differences.

\section{Cladosporium paris H.W. Shen, Y.X. Xu, H.Y. Su \& Z.L. Luo, sp. nov.}

- IndexFungorum $\underline{558844}$

- $\quad$ Species-ID Facesoffungi number: FoF 10539

\section{Material}

Holotype:

a. ScientificName: Cladosporium paris; kingdom: Fungi; phylum: Ascomycota; class:

Dothideomycetes; order: Capnodiales; family: Cladosporiaceae; genus: Cladosporium; locationRemarks: China, Yunnan Province, Dali, on diseased leaves of Paris polyphylla; year: 2020; habitat: leaf spots of Paris polyphylla; recordedBy: Yue-Xin Xu; collectionID: 2CL JD 18-2-1; collectionCode: Y-27

\section{Description}

Asexual morph: hyphomycetous (Fig. 3). Mycelium immersed and superficial, composed of septate, constricted at septa, unbranched, subhyaline, smooth hyphae, 2-6 $\mu \mathrm{m}$ wide. Conidiophores macronematous, 209-285 × 5-8 $\mu \mathrm{m}(\bar{x}=246.9 \times 6.5 \mu \mathrm{m}$, $\mathrm{n}=15)$, solitary or in small fascicles, erect to slightly flexuous, sometimes slightly geniculate, non-nodulose, sometimes subnodulose at the uppermost apex, unbranched, 0-6 septate, sometimes slightly constricted at septa, pale to olivaceousbrown, smooth or almost so. Conidiogenous cells cylindrical, sometimes geniculatesinuous, proliferation of sympodia with up to 5 conidiogenous loci, often crowded at the apex. Conidia $13-21 \times 7-12 \mu \mathrm{m}(\bar{x}=17 \times 9.7 \mu \mathrm{m}, \mathrm{n}=30)$, solitary or catenate, usually in simple chains, broadly ellipsoid-ovoid, rather pale, pale olivaceous or olivaceousbrown, verruculose, ends usually broadly rounded. Sexual morph: Undetermined.

Culture characteristics: Colonies on PDA attaining $21 \mathrm{~mm}$ diam. after $7 \mathrm{~d}, 40 \mathrm{~mm}$ diam. after $14 \mathrm{~d}$ and covering the whole Petridish after $30 \mathrm{~d}$, radially folded, furrowed, margin irregularly undulate; reverse olivaceous grey. 


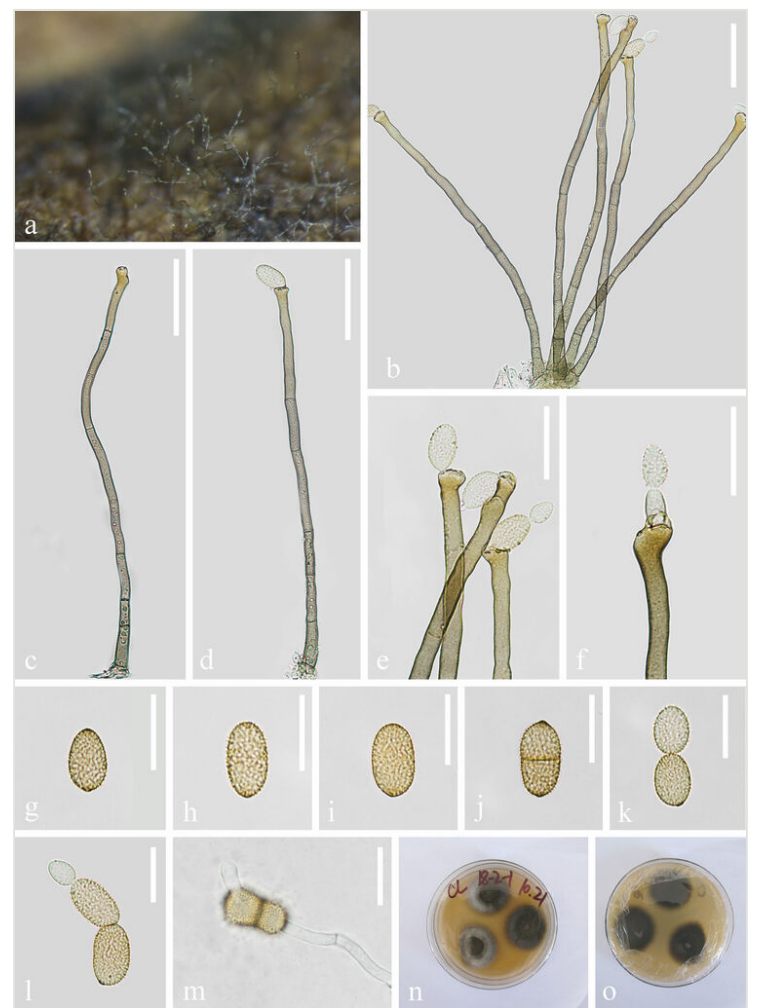

Figure 3. doi

Cladosporium paris (KUN-HKAS 121701, holotype). a Colonies on leaves; b,c Conidiophores; d Conidiophore with conidium; e,f Conidiogenous cells with conidia; g-I Conidia; $\mathbf{m}$ Germinating conidium; $\mathbf{n}, \mathbf{o}$ Culture on PDA from above and reverse. Scale bars: $\mathbf{b}-$ $\mathbf{d}=50 \mu \mathrm{m} ; \mathbf{e}, \mathbf{f}=30 \mu \mathrm{m} ; \mathbf{g}-\mathbf{m}=20 \mu \mathrm{m}$.

Material examined: China, Yunnan Province, Dali, on diseased leaves of Paris polyphylla, 16 October 2020, Y.X. Xu, Y-27. (KUN-HKAS 121701, holotype), ex-type living culture CGMCC $3.20623=$ KUNCC 21-10713.

\section{Etymology}

"paris" refers to the host genus, Paris.

\section{Distribution}

China, Yunnan Province, Dali, on diseased leaves of Paris polyphylla

\section{Notes}

Phylogenetic analysis showed that Cladosporium paris is closely related to $C$. floccosum (Fig. 1). Morphologically, our new isolate is distinguished from C. floccosum 
by its longer conidiophores (up to $285 \mu \mathrm{m}$ vs. up to $100 \mu \mathrm{m}$ ) and larger conidiogenous cells $(13-21 \times 7-12 \mu \mathrm{m}$ vs. $8-15 \times 6-8.5 \mu \mathrm{m})$. In addition, conidia of $C$. paris are 0-3 septate, while $C$. floccosum are $0-1$ septate (Sandoval-Denis et al. 2016). A comparison of the TEF1- $\alpha$ and ACT between C. paris (KUN-HKAS 121701) and $C$. floccosum (CBS 140463) reveals 3 and 16 nucleotide differences, respectively, which indicates that they are distinct taxa.

\section{Analysis}

\section{Phylogenetic analysis}

The combined ITS, TEF1- $\alpha$ and ACT dataset consisted of 161 sequences representing all genera of the Cladosporium with Toxicocladosporium irritans (CBS 185.58) and $T$. protearum (CBS 126499) as outgroup taxa. The best scoring RaxML tree with the final ML optimisation likelihood value of -24601.202740 is shown here (Fig. 1). The alignment comprised 1297 characters including gaps. The matrix had 775 distinct alignment patterns, with $15.38 \%$ undetermined characters or gaps. Estimated base frequencies were as follows: $\mathrm{A}=0.228337, \mathrm{C}=0.293636, \mathrm{G}=0.250484, \mathrm{~T}=0.227544$; substitution rates $\mathrm{AC}=$ 1.726214, $A G=3.618770, A T=1.752951, C G=1.098108, C T=5.802327, \mathrm{GT}=$ $1.000000 ;$ Tree-Length $=7.357731$.

Phylogenetic analyses of combined ITS, TEF1- $\alpha$ and ACT sequence data showed that the two new isolates of Cladosporium yunnanensis (KUN-HKAS 121704) and C. paris (KUNHKAS 121701) grouped with members of Cladosporium. Cladosporium yunnanensis (KUN-HKAS 121704) clustered with C. cladosporiordes (CBS 112388 and CBS 113738) and C. magnoliigena (MFLUCC 18-1559), but in an independent lineage with significant bootstrap (86 ML/1.00 PP). Cladosporium paris (KUN-HKAS 121701) formed a distinct lineage and sister to C. floccosum (CBS 140463) and basal to the genus with highlysupported value (94 ML/0.98 PP).

\section{Discussion}

In our study, based on the typical morphological features (Schubert et al. 2007, Zalar et al. 2007, Dugan et al. 2008, Bensch et al. 2010), Cladosporium yunnanensis and C. paris belong to the $C$. cladosporioides and $C$. herbarum species complex, respectively. The ITS sequences of the two new species are identical under the common barcode for fungi as previously reported studies for many other Cladosporium species (Bensch et al. 2010, Bensch et al. 2012, Marin-Felix et al. 2017). Therefore, multi-gene phylogenetic analysis (ITS, TEF1- $\alpha$ and ACT) can further prove the taxonomy of the two species in Cladosporium, which is consistent with the result by morphological features.

Cladosporium species are found as the dominant fungal genera in indoor and outdoor environments and are also important as saprobes and endophytes which have been screened from grains, fruits and chilled meat (Fradkin et al. 1987, Bullerman 2003, Hassan 
et al. 2021). However, Cladosporium yunnanensis and $C$. paris have been isolated from leaves of Paris polyphylla in Yunnan Province, China for the first time. Studies indicate that investigation on new hosts for fungi diversity would lead to the discovery of new fungal species and expand species resources (Hyde et al. 2018, Hyde et al. 2020). Certain Cladosporium species have been reported as producers of mycotoxin and to cause fungal allergies, particularly rhinitis and asthma. (Horner et al. 1995, Kurup 2003, Matheson et al. 2005, Simon-Nobbe et al. 2008, Mercier et al. 2013, Alwatban et al. 2014 , Segers et al. 2015). Both new species are isolated from diseased spots on plant leaves and many species of this genus are reported as plant pathogens, so they also have the potential to cause plant diseases. To determine whether these fungi are plant pathogens or have longterm adverse reactions on human health, pathogenicity determination and secondary metabolites of Cladosporium can be the focus of our future research.

\section{Acknowledgements}

This study is supported by the National Natural Science Foundation of China (Project ID: 31970021) and the Fungal Diversity Conservation and Utilization Innovation Team of Dali University (ZKLX2019213). Yue-Xin Xu thanks Rui Gu and Zheng-Quan Zhang for the help on collecting samples. We are grateful to and thank Ga-Heng Li and Hong-Yan Liu for their help on isolation, morphological examination, DNA extraction and PCR amplification.

\section{References}

- $\quad$ Abdollahzadeh J, Groenewald JZ, Coetzee MP, Wingfield MJ, Crous PW (2020) Evolution of lifestyles in Capnodiales. Studies in Mycology 95: 381-414. https://doi.org/ 10.1016/j.simyco.2020.02.004

- $\quad$ Adorisio S, Fierabracci A, Muscari I, Liberati AM, Cannarile L, Thuy TT, Sung TV, Sohrab H, Hasan CM, Ayroldi E, Riccardi C, Mazid A, Delfino DV (2019) Fusarubin and anhydro-fusarubin isolated from a Cladosporium species inhibit cell growth in human cancer cell lines. Toxins 11: 503. https://doi.org/10.3390/toxins11090503

- $\quad$ Alwatban MA, Hadi S, Moslem MA (2014) Mycotoxin production in Cladosporium species influenced by temperature regimes. Journal of Pure and Applied Microbiology 8: 4061-4069.

- $\quad$ Bensch K, Groenewald JZ, Starink-Willemse M, Andersen B, Sumerell BA, Shin HD (2010) Species and ecological diversity within the Cladosporium cladosporioides complex (Davidiellaceae, Capnodiales). Studies in Mycology 67 (1): 1-94. https://doi.org/10.1016/S0166-0616(14)60026-9

- $\quad$ Bensch K, Braun U, Groenewald JZ, Crous PW (2012) The genus Cladosporium. Studies in Mycology 72: 1-401. https://doi.org/10.3114/sim0003

- $\quad$ Bensch K, Groenewald JZ, Braun U, Dijksterhuis J, de Jesús Yáñez-Morales M, Crous PW (2015) Common but different: The expanding realm of Cladosporium. Studies in Mycology 82: 23-74. https://doi.org/10.1016/j.simyco.2015.10.001 
- $\quad$ Bensch K, Groenewald JZ, Meijer M, Dijksterhuis J, Jurjevic Z, Andersen B, Houbraken J, Crous PW, Samson RA (2018) Cladosporium species in indoor environments. Studies in Mycology 89: 177-301. https://doi.org/10.1016/i.simyco.2018.03.002

- Bezerra JD, Sandoval-Denis M, Paiva LM, Silva GA, Groenewald JZ, Souza-Mott CM, Crous PW (2017) New endophytic Toxicocladosporium species from cacti in Brazil, and description of Neocladosporium gen. nov. International Mycological Association Fungus 8 (1): 77-97. https://doi.org/10.5598/imafungus.2017.08.01.06

- Bullerman LB (2003) Encyclopedia of food sciences and nutrition. Spoilage (Fungi in Food - An Overview). Academic Press, 5511-5522 pp. [ISBN 012227055X] https://doi.org/10.1016/B0-12-227055-X/01129-9

- $\quad$ Chomnunti P, Hongsanan S, Aguirre-Hudson B, Tian Q, Persoh D, Dhami M, Xu JC, Liu XZ, Stadler M, Hyde K, Alias A (2014) The sooty moulds. Fungal Diversity 66: 1-36. https://doi.org/10.1007/s13225-014-0278-5

- $\quad$ Chung D, Kim H, Choi HS (2019) Fungi in salterns. Journal of Microbiology 57: 717-724. https://doi.org/10.1007/s12275-019-9195-3

- $\quad$ Crous PW, Braun U, Schubert K, Groenewald JZ (2007) The genus Cladosporium and similar dematiaceous hyphomycetes. Studies in Mycology 58: 1-253. https://doi.org/ 10.1016/S0166-0616(14)60115-9

- David JC (1997) A contribution to the systematics of Cladosporium. Revision of the fungi previously referred to Heterosporium. Mycological Papers 172: 1-157.

- Dugan FM, Schubert K, Braun U (2004) Check-list of Cladosporium names. Schlechtendalia 11: 1-103.

- Dugan FM, Braun U, Groenewald JZ, Crous PW (2008) Morphological plasticity in Cladosporium sphaerospermum. Persoonia 21: 9-16. https://doi.org/10.3767/003 $158508 \times 334389$

- $\quad$ Flannigan B, Samson RA, Miller JD (2002) Microorganisms in home and indoor work environments. Chemical Rubber Company [ISBN 9780429219535] https://doi.org/ 10.1201/9780203302934

- $\quad$ Fradkin A, Tobin RS, Tarlo SM, Tucic-Porretta M, Malloch D (1987) Species identification of airborne molds and its significance for the detection of indoor pollution. Japca 37: 51-53. https://doi.org/10.1080/08940630.1987.10466201

- $\quad$ Freitas MLR (2018) New species and new records of conidial fungi from submerged decayed leaves in Brazil. Universidade Federal de Viçosa URL: http://www.locus.ufv.br/ handle/123456789/21429

- Hassan A, Zeeshan M, Bhatti MF (2021) Indoor and outdoor microbiological air quality in naturally and mechanically ventilated university libraries. Atmospheric Pollution Research 12 (8): 101136. https://doi.org/10.1016/j.apr.2021.101136

- Hespanhol L, Vallio CS, Costa LM, Saragiotto BT (2019) Understanding and interpreting confidence and credible intervals around effect estimates. Brazilian Journal of Physical Therapy 23 (4): 290-301. https://doi.org/10.1016/j.bjpt.2018.12.006

- Heuchert B, Braun U, Schubert K (2005) Morphotaxonomic revision of fungicolous Cladosporium species (hyphomycetes). Schlechtendalia 13: 1-78.

- Horner WE, Helbling A, Salvaggio JE, Lehrer SB (1995) Fungal allergens. Clinical Microbiology Reviews Press 8: 161-179. https://doi.org/10.1128/CMR.8.2.161

- Hyde KD, Norphanphoun C, Chen J, Dissanayake AJ, Doilom M, Hongsanan S, Jayawardena RS, Jeewon R, Perera RH, Thongbai B, Wanasinghe DN, Wisitrassameewong K, Tibpromma S, Stadler M (2018) Thailand's amazing diversity: up 
to $96 \%$ of fungi in northern Thailand may be novel. Fungal Diversity 93 (1). https://doi.org/10.1007/s13225-018-0415-7

- Hyde KD, Jeewon R, Chen YJ, Bhunjun CS, Calabon MS, Jiang HB, Lin CG, Norphanphoun C, Sysouphanthong P, Pem D, Tibpromma S, Zhang Q, Doilom M, Jayawardena RS, Liu JK, Maharachchikumbura SS, Phukhamsakda C, Phookamsak R, Al-Sadi AM, Thongklang N, Wang Y, Gaforov Y, Jones EB, Lumyong S (2020) The numbers of fungi: is the descriptive curve flattening? Fungal Diversity 103 (1). https://doi.org/10.1007/s13225-020-00458-2

- Iturrieta-González I, García D, Gené J (2021) Novel species of Cladosporium from environmental sources in Spain. MycoKeys 77: 1. https://doi.org/10.3897/mycokeys. 77.60862

- Jayasiri SC, Hyde KD, Ariyawansa HA, Bhat J, Buyck B, Cai L, Dai YC, Abd-Elsalam KA, Ertz D, Hidayat I, Jeewon R, Jones EB, Bahkali AH, Karunarathna SC, Liu JK, Luangsa-Ard JJ, Lumbsch HT, Maharachchikumbura SS, Mckenzie EH, Moncalvo JM, Ghobad-Nejhad M, Nilsson H, Pang KL, Pereira OL, Phillips AJ, Raspé O, Rollins AW, Romero AI, Etayo J, Selçuk F, Stephenson SL, Suetrong S, Taylor JE, Tsui CK, Vizzini A, Abdel-Wahab MA, Wen TC, Boonmee S, Dai DQ, Daranagama DA, Dissanayake AJ, Ekanayaka AH, Fryar SC, Hongsanan S, Jayawardena RS, Li WJ, Perera RH, Phookamsak R, Silva NI, Thambugala KM, Tian Q, Wijayawardene NN, Zhao RL, Zhao Q, Kang JC, Promputtha I (2015) The faces of fungi database: fungal names linked with morphology, phylogeny and human impacts. Fungal Diversity 74: 3-18. https://doi.org/ 10.1007/s13225-015-0351-8

- Jayasiri SC, Hyde KD, Jones EB, McKenzie EH, Jeewon R, Phillips AJ, Bhat DJ, Wanasinghe DN, Liu JK, Lu YZ, Kang JC, Xu J, Karunarathna SC (2019) Diversity, morphology and molecular phylogeny of Dothideomycetes on decaying wild seed pods and fruits. Mycosphere 10 (1): 1-186. https://doi.org/10.5943/mycosphere/10/1/1

- Katoh K, Standley DM (2013) MAFFT multiple sequence alignment software version 7: improvements in performance and usability. Molecular Biology and Evolution 30 (4):

772-780. https://doi.org/10.1093/molbev/mst010

- Khan MI, Sohrab MH, Rony SR, Tareq FS, Hasan CM, Mazid MA (2016) Cytotoxic and antibacterial naphthoquinones from an endophytic fungus, Cladosporium sp. Toxicology Reports 3: 861-865. https://doi.org/10.1016/j.toxrep.2016.10.005

- Köhl h, Scheer C, Holb IJ, Masny S, Molhoek W (2015) Toward an integrated use of biological control by Cladosporium cladosporioides H39 in apple scab (Venturia inaequalis) management. Plant Disease 99: 535-543. https://doi.org/10.1094/PDIS08-14-0836-RE

- $\quad$ Kurup VP (2003) Fungal allergens. Current Allergy and Asthma Reports 3 (5): 416. https://doi.org/10.1007/s11882-003-0078-6

- $\quad$ Link HF (1816) Observationes in ordines plantarum naturales. Dissertatio II, sistens nuperas de Mucedinum et Gastromycorum ordinibus observationes. Der Gesellschaft Naturforschender Freunde zu Berlin Magazin für die Neuesten Entdeckungen in der Gesammten Naturkunde 7: 25-45.

- Marin-Felix Y, Groenewald JZ, Cai L, Chen Q, Marincowitz S, Chen Q, Marincowitz S, Barnes I, Bensch K, Braun U, Camporesi E, Damm U, de Beer ZW, Dissanayake A, Edwards J, Giraldo A, Hernández-Restrepo M, Hyde KD, Jayawardena RS, Lombard L, Crous PW (2017) Genera of phytopathogenic fungi: GOPHY 1. Studies in Mycology 86: 99-216. https://doi.org/10.1016/j.simyco.2017.04.002 
- Matheson MC, Abramson MJ, Dharmage SC, Forbes AB, Raven JM, Thien FC, Walters $\mathrm{EH}$ (2005) Changes in indoor allergen and fungal levels predict changes in asthma activity among young adults. Clinical \& Experimental Allergy 35: 907-913.

https://doi.org/10.1111/j.1365-2222.2005.02272.x

- $\quad$ Mercier E, Peters IR, Billen F, Battaille G, Clercx C, Day MJ, Peeters D (2013) Potential role of Alternaria and Cladosporium species in canine lymphoplasmacytic rhinitis. Journal of Small Animal Practice 54 (4): 179-183. https://doi.org/10.1111/jsap.12049

- $\quad$ Nylander JA (2004) MrModeltest v2.2 Program distributed by the author. Evolutionary Biology Centre, Uppsala University, Uppsala. URL: https://www.researchgate.net/ publication/285805344

- $\quad$ Persoon CH (1794) Dispositio methodica fungorum. Romers Neues Mag Bot 1: 81-128.

- $\quad$ Rambaut A, Drummond A (2008) FigTree: Tree figure drawing tool, version 1.2. 2. Institute of Evolutionary Biology, University of Edinburgh URL: http://tree.bio.ed.ac.uk/ software/figtree/

- $\quad$ Ronquist F, Huelsenbeck JP (2003) MrBayes 3: Bayesian phylogenetic inference under mixed models. Bioinformatics 19: 1572-1574. https://doi.org/10.1093/bioinformatics/ $\underline{\operatorname{btg} 180}$

- $\quad$ Rosado AW, Custodio FA, Pinho DB, Ferreira AP, Pereira OL (2019) Cladosporium species associated with disease symptoms on Passiflora edulis and other crops in Brazil, with descriptions of two new species. Phytotaxa 409 (5): 239-260. https://doi.org/ 10.11646/phytotaxa.409.5.1

- Sandoval-Denis M, Sutton DA, Martin-Vicente A, Cano-Lira JF, Wiederhold N, Guarro J, Gené J (2015) Cladosporium species recovered from clinical samples in the United States. Journal of Clinical Microbiology 53: 2990-3000. https://doi.org/10.1128/JCM. 01482-15

- Sandoval-Denis M, Gené J, Sutton DA, Wiederhold NP, Cano-Lira JF, Guarro J (2016) New species of Cladosporium associated with human and animal infections. Persoonia: Molecular Phylogeny and Evolution of Fungi 36: 281. https://doi.org/10.3767/00315 8516X691951

- $\quad$ Schubert K (2005) Morphotaxonomic revision of foliicolous Cladosporium species (Hyphomycetes). Martin-Luther-University, Halle-Wittenberg https://doi.org/ $10.25673 / 3679$

- $\quad$ Schubert K, Groenewald JZ, Braun U, Dijksterhuis J, Starink M, Hill CF, Zalar P, de Hoog GS, Crous PW (2007) Biodiversity in the Cladosporium herbarum complex (Davidiellaceae, Capnodiales), with standardisation of methods for Cladosporium taxonomy and diagnostics. Studies in Mycology 58: 105-156. https://doi.org/10.3114/ sim.2007.58.05

- $\quad$ Segers FJ, Meijer M, Houbraken J, Samson RA, Wösten HA, Dijksterhuis J (2015) Xerotolerant Cladosporium sphaerospermum are predominant on indoor surfaces compared to other Cladosporium species. Public Library of Science One 10: 145-150. https://doi.org/10.1371/journal.pone.0145415

- Silvestro D, Michalak I (2012) raxmIGUI: a graphical front-end for RAxML. Organisms Diversity \& Evolution 12: 335-337. https://doi.org/10.1007/s13127-011-0056-0

- Simon-Nobbe B, Denk U, Pöll I, Rid R, Breitenbach M (2008) The spectrum of fungal allergy. International Archives of Allergy and Immunology 14: 58-86. https://doi.org/ $10.1159 / 000107578$ 
- $\quad$ Temperini CV, Pardo AG, Pose GN (2018) Diversity of airborne Cladosporium species isolated from agricultural environments of northern Argentinean Patagonia: molecular characterization and plant pathogenicity. Aerobiologia 34: 227-239. https://doi.org/ 10.1007/s10453-018-9509-7

- $\quad$ Tibpromma S, Mortimer PE, Karunarathna SC, Zhan F, Xu J, Promputtha I, Yan K (2019) Morphology and multi-gene phylogeny reveal Pestalotiopsis pinicola sp. nov. and a new host record of Cladosporium anthropophilum from edible pine (Pinus armandii) seeds in Yunnan Province, China. Pathogens 8 (4): 285. https://doi.org/10.3390/ pathogens8040285

- Velázquez-Jiménez Y, Hernández-Castro R, Romero-Romero L, Salas-Garrido CG, Martínez-Chavarría LC (2019) Feline phaeohyphomycotic cerebellitis caused by Cladosporium cladosporioides-complex: Case report and review of literature. Journal of Comparative Pathology 170: 78-85. https://doi.org/10.1016/j.jcpa.2019.05.011

- Zalar PD, De Hoog GS, Schroers HJ, Crous PW, Groenewald JZ, Gunde-Cimerman N (2007) Phylogeny and ecology of the ubiquitous saprobe Cladosporium sphaerospermum, with descriptions of seven new species from hypersaline environments. Studies in Mycology 58: 157-183. https://doi.org/10.3114/sim.2007.58.06

- Zhou YB, Chen P, Sun TT, Wang XJ, Li DM (2016) Acne-like subcutaneous phaeohyphomycosis caused by Cladosporium cladosporioides: a rare case report and review of published literatures. Mycopathologia 181: 567-573. https://doi.org/10.1007/ s11046-016-9995-5

- Zimowska B, Becchimanzi A, Krol ED, Furmanczyk A, Bensch K, Nicoletti R (2021) New Cladosporium species from normal and galled flowers of Lamiaceae. Pathogens 10: 369. https://doi.org/10.3390/pathogens 10030369 